\title{
OS DETERMINANTES DO ÍNDICE DE DESEMPENHO ACADÊMICO DOS ESTUDANTES DO SEMIÁRIDO POTIGUAR
}

\author{
B. A. VIEIRA ${ }^{*}$, L. NOGUEIRA e W. P. S. F. SOUZA \\ Universidade Federal Rural do Semiárido - Campus Pau dos Ferros - RN \\ Universidade Federal do Rio Grande do Sul \\ biancaalencaruna@hotmail.com ${ }^{*}$ \\ Submetido08/04/2016 - Aceito f03/02/2017
}

DOI: $10.15628 /$ holos.2017.4388

\section{RESUMO}

Este estudo tem como principal objetivo mensurar os principais determinantes do Índice de Rendimento Acadêmico dos Estudantes do Semiárido Potiguar. Para tanto, adotou-se três metodologias associadas a um questionário socioeconômico. Os principais resultados possibilitam ao menos fazer três inferências: i) participação significativa das condições sociais no desempenho educacional; ii) substancial diferencial de esforço para o aluno com baixo background social atingir a média, e a partir dessa, o esforço parece dominar consideravelmente as condições sociais desfavoráveis; e, por fim iii) há fortes evidências de efeito tratamento para as variáveis de esforço.

PALAVRAS-CHAVE: Índice de Rendimento Acadêmico, Desigualdade de Oportunidades Educacionais, Efeito Tratamento.

\section{THE DETERMINATIONS OF THE ACADEMIC PERFORMANCE INDEX OF STUDENTS OF THE SEMIARID POTIGUAR}

\begin{abstract}
This study has as main objective to measure the main determinants of the Academic Income Index of the Students of the Semiarid Potiguar. For that, three methodologies associated to a socioeconomic questionnaire were adopted. The main results allow at least three inferences: i) significant participation of
\end{abstract}

social conditions in educational performance; li) substantial differential of effort for the student with low social background to reach the average, and from there, the effort seems to dominate considerably unfavorable social conditions; And finally iii) there is strong evidence of treatment effect for effort variables.

KEYWORDS: Academic Performance Index, Inequality of Educational Opportunities, Treatment Effect. 


\section{INTRODUÇÃO}

Em geral, estudos econômicos, políticos e filosóficos encontram fortes evidências de que a escolaridade é um fator crucial na determinação do sucesso econômico e educacional individual. A esse respeito, ver, por exemplo, Dworkin (1981) e Arneson (1989), que defendem as diferenças na renda atreladas a preferências individuais, e autores como Roemer (1998), que tratam do conceito de desigualdade de oportunidades ${ }^{1}$.

Seguindo esse último enfoque, os resultados econômicos individuais (renda, educação) dependem de variáveis de responsabilidade, denominadas de esforço, e variáveis sobre as quais os indivíduos não têm responsabilidade, definidas como circunstâncias. Dessa forma, vários pesquisadores argumentam que apenas a desigualdade devido a variáveis de não responsabilidade (também denominada de desigualdade injusta) é socialmente indesejável. Essa definição se aplica adequadamente aos determinantes do nível educacional, dado que é sabido que os antecedentes familiares e as condições do local de nascimento exercem um efeito considerável sobre tais resultados, assim como características sob o controle dos próprios indivíduos, tais como motivação, esforço, determinação, entre outras.

A qualidade da educação é enxergada consensualmente como fator imprescindível no desenvolvimento social de qualquer região, seja via aumento da produtividade e/ou elevação do nível médio de salários da população. Entretanto, apesar da importância atribuída à qualidade educacional, em todos os setores da sociedade, o que se observa na realidade é um nível de ações ainda bastante insipiente para a obtenção de um sistema educacional público de qualidade, especialmente nas nações em desenvolvimento.

Nesse ensejo, Daude (2011), relata que a promoção de uma educação de qualidade é um instrumento básico para o bem estar social. Em outras palavras, o estudo descreve que a oferta de uma educação de qualidade para todos é uma necessidade insubstituível, especialmente para equalização das oportunidades entre aqueles menos favorecidos circunstancialmente.

Em outro estudo, Black, Devereux e Salvanes (2005), apontam dois princípios essenciais que definem a qualidade de um sistema educacional. $\mathrm{O}$ primeiro institui que o desenvolvimento cognitivo dos alunos é a principal meta de qualquer sistema, onde o sucesso alcançado pelos discentes é sem dúvida um forte indicador de sua qualidade. Já o segundo, assinala o papel da educação na promoção de valores compartilhados e no desenvolvimento criativo e emocional. Nesse caso, a obtenção desse objetivo é bem mais ampla, inclusive pela complexidade de análise e mensuração.

Adicionalmente, o estudo advoga que em qualquer sistema educacional devem-se haver determinadas condições para que isso se configure. Por exemplo, todos os indivíduos precisam frequentar instituições de ensino de qualidade, independentemente do seu ambiente de oportunidades. Além disso, a sociedade deve prestigiar o mérito, de forma que, o talento e as habilidades individuais prevaleçam sempre, pois sem essas garantias os retornos dos investimentos educacionais são insuficientes, especialmente, para aqueles mais vulneráveis. 0

\footnotetext{
${ }^{1}$ A seção 2.1 tratará sobre a definição da desigualdade de oportunidades.
} 
estudo conclui que somente assim consegue-se reduzir permanentemente a persistência intergeracional da educação.

Diante disso, nos últimos anos, estudos que contemplam resultados de desempenho educacionais vêm ganhando destaque frente aos que utilizam a variável anos de estudo como proxy de nível de educação, pois esta última não garante necessariamente a qualidade do sistema de ensino avaliado $^{2}$. Isso ocorre, essencialmente, em função de dois aspectos fundamentais: i) a heterogeneidade existente - qualidade educacional - entre escolas, regiões, etc.; e ii) a possível captação dos determinantes de transmissão educacional.

Posto isto, origina-se um importante questionamento quanto ao índice de desempenho educacional dos alunos das universidades federais brasileiras, em especial, daquelas localizadas em regiões mais pobres: o que determina o índice de desempenho educacional dos alunos de instituições de ensino superior com estas características? Assim sendo, este estudo tem como principal objetivo investigar quais são os fatores cruciais na determinação do desempenho educacional dos estudantes da Universidade Federal Rural do Semiárido - UFERSA - no campus de Pau dos Ferros, Estado do Rio Grande do Norte. A seleção desse campus como estudo de caso ocorre em especial por dois fatores: i) a maior parte dos alunos tem origem familiar no semiárido; e ii) o alto índice de migrantes de estudantes do semiárido paraibano e cearense na composição dos discentes desse campus.

Para tanto, a estratégia empírica utilizada consiste de três etapas para captar tais fatores que determinam o desempenho educacional dos estudantes supracitados. Em primeiro lugar usa-se a função de produção educacional, seguindo Hanushek $(1970,1979)$ para estimar os fatores que afetam o rendimento escolar. Em seguida é aplicado um modelo bivariado para caracterizar o vetor de escolhas e resultados dos indivíduos, e por fim, o método de efeito de tratamento seguindo Cameron e Trivedi (2005) é utilizado para verificar o impacto da diferença de background familiar entre os estudantes.

Esse estudo segue a seguinte estrutura além dessa introdução. Na seção dois apresentamse aspectos teóricos sobre a desigualdade de oportunidades e alguns trabalhos voltados para o problema investigado. A terceira seção é reservada a explanar a estratégia empírica proposta, como também a análise sumária dos dados. A quarta seção é destinada a reportar os principais resultados observados e, por fim, na quinta seção são feitas as considerações finais.

\section{REVISÃO BIBLIOGRÁFICA}

Esta seção apresenta uma breve revisão de literatura sobre o tema abordado. Em primeiro lugar, na seção 2.1 serão destacados os conceitos teóricos associados à literatura da desigualdade de oportunidades ou desigualdade injusta. Em seguida, são apresentados alguns trabalhos sobre os determinantes do desempenho educacional, a partir da utilização de diferentes métodos de estimação.

\footnotetext{
${ }^{2}$ Para mais detalhes sobre avaliação de sistema educacional via medidas de desempenho ver Figueiredo, Nogueira e Santana (2014); Anshenfelter e Rouse (1998); Ferreira e Gignoux, (2011), entre outros.
} 


\subsection{Desigualdade de Oportunidades: Aspectos Teóricos}

A abordagem tradicional da desigualdade de oportunidades considera que o resultado econômico de um indivíduo é fruto de fatores de circunstância, tais como backgroud familiar, raça, gênero, entre outros; e de esforço, associado às variáveis cujos indivíduos possuem controle (Roemer, 1998). O conceito de desigualdade usualmente presente em trabalhos empíricos está relacionado à igualdade de resultados como parâmetro de justiça, desconsiderando os fatores que levaram os indivíduos a possuírem determinado nível de renda. A partir de discussões filosóficas mais recentes surge a ideia de Igualdade de Oportunidades (Rawls, 1971; Roemer, 1998), como contraponto a essa visão igualitária, presente em trabalhos como o de Dworkin (1981) e Arneson (1989). Dessa forma, apenas a desigualdade oriunda de variáveis de circunstância é socialmente indesejável. Portanto, equalizar as oportunidades significa corrigir as circunstâncias desiguais e manter as diferenças de esforço inalteradas.

Contudo, embora o conceito de igualdade de oportunidades seja simples, sua operacionalização carrega alguns desafios importantes, destacando-se, por exemplo, a definição da variável de esforço. Em razão disso, alguns trabalhos, como Fleurbaey (1995), optam por considerar o esforço uma variável não observável. Já outros estudos, como Borguingnonet al (2007) e Annegues, Souza e Figueiredo (2015), procuram mensurá-lo utilizando alguma proxy, tal como escolaridade ou condição no mercado de trabalho. Saber se essas variáveis refletem de fato a escolha dos agentes é outro desafio das aplicações empíricas da teoria. É possível argumentar ainda que o esforço sofre problemas de endogeneidade, sobretudo com relação às circunstâncias.

Sendo assim, o presente estudo optou por usar o esforço como uma variável observável, mensurada a partir de proxies para saber se o aluno é repetente, migrante e a quantidade de horas de estudo semanal ${ }^{3}$. A utilização dessa abordagem, embora carregue problemas teóricos e empíricos, tem por objetivo identificar o efeito que fatores como background familiar, condicionado a determinado nível de esforço, exercem no resultado educacional dos indivíduos pesquisados.

\subsection{Revisão de literatura}

Nessa seção são apresentados alguns estudos voltados para o tema em questão. Por exemplo, Figueiredo e Nogueira (2014), utilizando dados do PISA 2012 investigam três relevantes aspectos sobre o desempenho educacional dos jovens que participaram do exame. Na primeira parte, os resultados evidenciam baixa transmissão intergeracional da educação nos países em desenvolvimento, em especial, aos países da América do Sul. Na segunda etapa, o estudo encontra fortes evidências da presença de efeitos indiretos das circunstâncias sobre o resultado, e, por fim, o estudo revela um efeito tratamento - educação parental - significativamente menor nos países desenvolvidos.

Em outro estudo, Figueiredo, Nogueira e Santana (2014), analisam a influência das circunstâncias no desempenho dos alunos que prestaram o ENEM 2010. Os resultados encontrados permitem observar que a probabilidade dos indivíduos que possuem baixo background social atingir nota superior à média nacional é quatro vezes menor comparado aos

\footnotetext{
${ }^{3}$ Todas as variáveis utilizadas serão descritas na seção 3.4 .
} 
indivíduos que possuem alto background social. Constatou-se também, que os alunos que moram nas regiões Norte e Nordeste apresentam menores desempenhos educacionais comparado com as outras regiões do Brasil. Além disso, foi possível averiguar que os efeitos indiretos transmitidos em relação à educação da mãe são 2,36 vezes mais elevadosdo que os efeitos diretos.

Por outro lado, Justo, Leite e Netto (2011), verificam os determinantes para a mobilidade intergeracional da educação no Brasil, buscando principalmente comparar entre as regiões Sudeste e Nordesteno período de 1992 a 2009, utilizando dados fornecidos do Instituto Brasileiro de Geografia e Estatística - IBGE. Os resultados tornam possível observar que a distribuição da educação do Brasil como um todo melhorou e que os anos de estudo evoluíram positivamente. Porém, verificou-se uma disparidade entre a educação do Nordeste e do Sudeste. Adicionalmente, averiguou-se que no período estudado a mobilidade educacional é maior no Sudeste. Especificamente, concluíram que a mobilidade intergeracional da educação é influenciada pela cor do indivíduo e dos pais, raça, sexo, nível educacional dos pais e renda per capita da família.

Netto Junior, Ramalho e Aragón (2013), buscam avaliar os determinantes da mobilidade intergeracional de renda no Brasil. Além disso, objetivam relacioná-la com a mobilidade intergeracional de educação. Para tanto, empregou-se dados da Pesquisa Nacional de Amostra de Domicílios - PNAD - para os anos de 1991 e 2000. Os resultados informam que uma família com estrutura biparental, ou seja, com o chefe e o cônjuge, influencia positivamente na mobilidade intergeracional de renda, quando comparado com uma família monoparental. Por outro lado, famílias monoparentais com seu chefe do sexo feminino exercem melhor influência quando comparadas as famílias com o chefe masculino. Além da estrutura familiar, a escolaridade dos pais, diferença de idade dos pais, cor do filho e pai/mãe e localização regional da família afetam na mobilidade educacional e de renda. Além disso, observou que a mobilidade educacional é intimamente relacionada à mobilidade de renda.

Por sua vez, Diaz (2013) analisa a desigualdade de oportunidades observada no ensino médio do Brasil. Para tanto, utilizou os dados do Sistema de Avaliação da Educação Básica - SAEB - referentes ao período de 1995 a 2005, quanto ao desempenho dos alunos do último ano do ensino médio em língua portuguesa e matemática. Foi possível perceber que a desigualdade de oportunidades foi maior na área de matemática do que em língua portuguesa. Além disso, verificou que a desigualdade por tipo da escola - pública e privada - tende a permanecer no mesmo padrão no período observado. Porém, constatou uma queda da desigualdade de oportunidades em relação às duas áreas analisadas nas escolas privadas em 2005. Entretanto, nas escolas públicas, os níveis de desigualdades permaneceram estabilizados no mesmo período.

Firpo, Ponczek e Possebom (2014), investigam se informações sobre o desempenho em provas - ENEM - afetam a mensalidade das escolas privadas. O estudo utiliza dados longitudinais no nível da escola e estima o efeito da publicação das notas do ENEM sobre a mensalidade escolar para três diferentes níveis: fundamental I,fundamental II e médio. Os resultados obtidos assinalam que o desempenho médio das escolas é positivamente correlacionado com mensalidades para todos os níveis educacionais, sendo maior para o ensino médio. Além disso, essa correlação é maior para escolas cujo desempenho absoluto era menos previsível em 2006. Segundo o estudo, tal característica indica que esse efeito acontece em função da publicação de nova e valiosa informação sobre o desempenho absoluto das escolas. 
Por sua vez, Machado e Szerman (2015) analisam os efeitos da introdução do SISU sobre a migração e a evasão estudantil, através da utilização dos microdados do Censo da Educação Superior. Os resultados apontam que a adoção do SISU possui correlação de 3,8\% com a migração observada entre municípios e 1,6\% entre estados. Adicionalmente, o estudo encontra um efeito de $4,5 \%$ sobre a evasão, apontando os custos decorrentes da migração e as condutas escolhidas pelos estudantes como principais determinantes da evasão dos alunos.

Romanello, Sawyer e Gonçalves (2013), investigam a participação da formação educacional individual sobre o tipo de entrada no mercado de trabalho. Para tanto, utilizam dados da Pesquisa Mensal de Emprego - PME - referente ao período de 2008 a 2013, associada à metodologia decompeting-risks regression. Os resultados apontam que os indivíduos que possuem nível médio apresentam uma probabilidade de trânsito para empregos formais variando entre $91 \%$ a $94 \%$ acima dos que obtiveram apenas o primeiro ciclo do ensino fundamental. Além disso, o estudo informa que indivíduos com no mínimo formação superior têm uma probabilidade a mais de trânsito para empregos formais variando entre $115 \%$ a $140 \%$ comparado aos que obtiveram apenas o primeiro ciclo do fundamental. Adicionalmente, observa-se que quão maior o grau educacional individual menor a possibilidade de trânsito para empregos informais e à situação de não trabalho.

Feita esse breve revisão acerca dos estudos que analisam a influência do nível educacional no contexto da desigualdade de oportunidades, a próxima seção trás os aspectos metodológicos empregados para alcançar o objetivo proposto no presente estudo.

\section{METODOLOGIA}

Este estudo adota três métodos como estratégia empírica, considerando ambos os aspectos quantitativos e qualitativos. Para tanto, são utilizados os dados de um questionário socioeconômico - probabilístico - coletado aleatoriamente em entrevista de 188 alunos pertencentes ao Campus da UFERSA em Pau dos Ferros/RN - de um total de 742 alunos - entre todos os cursos de graduação ofertados. Respectivamente, utilizam-se os seguintes mecanismos metodológicos: o modelo de Função de Produção Educacional de Hanuschek (1970, 1979), doravante denominada FPE, em conjunção com o instrumental desenvolvido por O'Neill, Sweetman e van DeGaer (2001). Adicionalmente, aplica-se a metodologia de efeito tratamento seguindo literalmente Cameron e Trivedi(2005).

\subsection{Função de Produção Educacional}

A FPE analisa o modo como os fatores do processo educacional podem afetar os resultados educacionais dos indivíduos, servindo de respaldo para a escolha dos principais determinantes educacionais, e com isso proporcionando um guia para a aplicação de políticas públicas. De acordo Hanuschek $(1970,1979)$, a FPE é dada por:

$$
A_{i t}=g\left(F_{i}^{t}, P_{i}^{t}, I_{i} S_{i}^{t}\right)
$$

Em que $A_{i t}$ é o vetor educacional realizado pelo i-ésimo estudante no tempo t; $F_{i t}$ é o vetor de características individuais e familiares do i-ésimo estudante acumulado no período t; $P_{i t}$ é o vetor do corpo discente (influências de pares), ou seja, variáveis socioeconômicas e 
background familiar de outros estudantes da escola acumulados no período t; $I_{i}$ é o vetor de dotações iniciais do i-ésimo indivíduo; e $S_{i t}$ é o vetor de insumos escolares relevantes para o iésimo estudante acumulados no período $t$.

Cabe aqui ressaltar um aspecto relevante para a estimação de (1), qual seja, possíveis problemas de omissão de variáveis, por exemplo, a habilidade do indivíduo. Isto porque é uma variável de difícil mensuração e a sua omissão torna os regressores do Ordinary Least Square (OLS) endógenos, além de deparar-se com problemas de simultaneidade. Um exemplo clássico seria o observado entre a renda dos pais e habilidade dos filhos. Pois, quanto mais educado o indivíduo, em média, maior o nível de habilidade dos filhos. De modo semelhante, quanto maior a habilidade, maior o nível de renda. Nesse sentido, a fim de superar esses percalços utiliza-se a metodologia de efeito tratamento, seguindo o exposto em Cameron e Trivedi,(2005). Porém, anteriormente, emprega-se uma estratégia idêntica a proposta por O'Neill, Sweetman e van DeGaer (2001), com o propósito de mensurar a quantidade de esforço - em termos de probabilidades - empregado por indivíduos com características sociais familiares - tipos completamente distintas. Isto é, comparam-se indivíduos socialmente vulneráveis, mas com uma situação social oposta. Faz-se um breve resumo a seguir desses métodos.

\subsection{Modelo Bivariado}

Segundo O'Neill, Sweetman e van DeGaer (2001), e admitindo que não há políticas compensatórias, o conjunto de oportunidades do estudante pode ser representado por $S_{-} x$, onde o mesmo é determinado por um vetor de características de não responsabilidade, $\mathrm{x}$. Esses pressupostos fazem com que o resultado individual obtido dependa do nível de esforço individual empregado, ou seja, das características de responsabilidade individual condicionado ao conjunto de oportunidade que o indivíduo possui.

Diante disso, os estudantes podem optar por diferentes escolhas e diferentes resultados. Estes podem ser resumidos por $z=y[e, x]$, em que $z$ representa a utilidade ou desempenho alcançado, no caso da pesquisa o Índice de Rendimento Acadêmico ao longo do tempo; e (e) representa o esforço individual empregado. Assume-se, também, que a função de distribuição de (e) é contínua e aceita duas suposições chaves:

- SINC (Strictlylncreasing): $\mathrm{z}=\mathrm{y}[\mathrm{e}, \mathrm{x}]$ é estritamente crescente em e. Isto é, quanto maior o nível de esforço maior a utilidade resultante. Definindo $\mathrm{F}_{\mathrm{z}}^{*}(\mathrm{z} \mid \mathrm{x})$ e $\mathrm{F}_{\mathrm{e}}^{*}(\mathrm{e} \mid \mathrm{x})$ a função de distribuição acumulada - FDA - respectiva $(\mathrm{z}, \mathrm{e})$, ambos condicionadas a $\mathrm{X}$, respectivamente. Assim, pode-se expressar SINC por:

$$
\mathrm{F}_{\mathrm{z}}^{*}(\mathrm{y}[\mathrm{e}, \mathrm{x}] \mid \mathrm{x})=\mathrm{F}_{\mathrm{e}}^{*}(\mathrm{e} \mid \mathrm{x})
$$

A expressão em (2) revela que o nível de esforço estudantil (e) condicionado ao seu tipo conjunto de oportunidades - será menor que $\alpha$ - th percentil da distribuição do seu esforço se, e somente se, o resultado for inferior a $\alpha$ - th percentil.

- IND (independente): $\mathrm{F}_{\mathrm{e}}^{*}(\mathrm{e} \mid \mathrm{x})$ é independente de $\mathrm{x}$. Esta premissa implica que não são assumidas diferenças na FDA do esforço entre tipos diferentes, ou seja, entre indivíduos 
com diferentes características de não responsabilidade, pertencentes ao mesmo percentil. Dito de outro modo, os indivíduos não podem ser responsáveis pelo nível de esforço empregado, caso estes dependam das circunstâncias aos quais estivessem inseridos. A Equação (2) associada a condição de independência acima - IND - resulta no Axioma de Identificação de Roemer (RIA).

- RIA (Roemer's Identification Axion): $\mathrm{F}_{\mathrm{z}}^{*}(\mathrm{y}[\mathrm{e}, \mathrm{x}] \mid \mathrm{x})=\mathrm{F}_{\mathrm{e}}^{*}(\mathrm{e} \mid \mathrm{x}) \Rightarrow \mathrm{e}^{\prime}=\mathrm{e}^{\prime \prime}$. De forma resumida, a RIA sugere que dois indivíduos - estudantes - com distintos conjuntos de oportunidades, isto é, circunstâncias, mas com o mesmo percentil da distribuição dentro do seu tipo, exercem o mesmo nível de esforço.

Assumindo tais pressupostos, define-se $\pi=\mathrm{F}_{\mathrm{z}}^{*}(\mathrm{z} \mid \mathrm{x})$ como a FDA do resultado de $\mathrm{z}$ condicionado as características de não responsabilidade, $\mathrm{x}$. Analogamente, assume-se que esta função é estritamente crescente em $\mathrm{z} . \mathrm{F}_{\mathrm{z}}^{-1}(\pi \mid \mathrm{x})$. E expressa o resultado obtido - IRA dos alunos da UFERSA - pelo indivíduo do tipo x e que estava em $100 * \pi$ - th percentil da FDA do resultado dentro do seu tipo. De acordo com a RIA, $F_{z}^{-1}(\pi \mid x)$ equivale a observar $y[p, x]$. Entretanto, $\mathrm{F}_{\mathrm{z}}^{-1}(\pi \mid \mathrm{x})$ fornecerá informações das características de responsabilidade e não responsabilidade dos indivíduos.

Segundo os autores, assim é possível desenhar o resultado - desempenho educacional com uma função de $\pi \in[0,1]$ para diferentes valores de $\mathrm{x}$. Sendo o conjunto de oportunidades para um particular tipo de $\mathrm{x}$ determinado por alguns resultados do tipo $\mathrm{x}$, e podendo ser obtido ao variar suas características de responsabilidade e ou $\pi$. Desse modo, o conjunto de oportunidades do estudante do tipo x será:

$$
\mathrm{S}_{\mathrm{x}}=(\mathrm{z}, \pi) \in\left(\mathbb{R}^{+} \times[0,1]\right) \mathrm{z}=\mathrm{F}_{\mathrm{z}}^{*-1}(\pi \mid \mathrm{x})
$$

Onde $\mathbb{R}^{+}$representa o conjunto dos números reais não negativos. Portanto, $\operatorname{seF}_{\mathrm{z}}^{*-1}$ estiver disponível, pode-se descrever o conjunto de oportunidades para diferentes tipos de indivíduos e, também, qual a extensão das opções diferentes ou níveis de esforço que produzem diferentes resultados. Adicionalmente, assume-se que a sociedade é responsável pela escolha dos componentes de $\mathrm{x}$. Além disso, considera-se que $\mathrm{x}$ é uma variável multidimensional composta por elementos como raça, sexo, background familiar e habilidade inata. Todavia, as curvas de densidade acumuladas do resultado de $\mathrm{z}$ condicionada às características de não responsabilidade de $\mathrm{x}$, serão inferidas a partir da modelagem kernel bivariada.

\subsection{Modelo de Efeito de Tratamento}

Seguindo Cameron e Trivedi (2005), é definida a diferença $\Delta$ de resultado entre o estado de tratado e não tratado conforme a variável de interesse em questão. Assim, expresso:

$$
\Delta=y_{1}-y_{0}
$$


De modo que, permitisse condicionar $x$. Uma vez que, não é possível observar diretamente o mesmo indivíduo em ambos os estados no mesmo período de tempo, como por exemplo, certo indivíduo sendo soro positivo e não positivo para hepatite. Assim, os valores da população, quanto ao efeito do tratamento e efeito do tratamento sobre os tratados são definidos respectivamente como:

$$
\begin{gathered}
\mathrm{ATE}=\mathrm{E}[\Delta], \\
\mathrm{ATET}=\mathrm{E}[\Delta \mid D=1],
\end{gathered}
$$

Analogamente,

$$
\begin{gathered}
\widehat{A T E}=\frac{1}{N} \sum_{i=1}^{N} \Delta_{i} \\
\widehat{\operatorname{ATET}}=\frac{1}{N_{T}} \sum_{i=1}^{N_{T}}\left[\Delta_{i} \mid D_{i}=1\right],
\end{gathered}
$$

Onde $N_{T}=\sum_{i=1}^{N} D_{i}$. Para cada um dos dois casos acima o cálculo é simples se $\Delta_{i}$ é observado. Contudo, a obtenção das estimações não é direta, porque ambas as expressões em (7) e (8) tem um componente não observado, e, portanto, deve ser estimado, o que exige alguns pressupostos. De acordo com Heckman e Vytlacil (2002), o valor apurado ATE é relevante quando o tratamento se aplica a qualquer indivíduo da população. De forma que, é admissível considerar o efeito hipotético do tratamento para um indivíduo elegido aleatoriamente entre a população. Por sua vez, a estimação proposta em ATET é apropriada quando objetiva-se apurar o efeito médio de tratamento para os tratados. Isto é, o problema de avaliação do tratamento considera o ganho médio de participação dadas as características em $x$. Assim, tem-se:

$$
\begin{gathered}
\mathrm{ATE}=[\Delta \mid X=x] \\
\mathrm{ATE}=\left[y_{1}-y_{0} \mid X=x\right] \\
\mathrm{ATE}=\mathrm{E}\left[y_{1} \mid X=x\right]-\mathrm{E}\left[y_{0} \mid X=x\right] \\
\mathrm{ATE}=\mathrm{E}\left[y_{1} \mid x, D=1\right]-\mathrm{E}\left[y_{0} \mid x, D=0\right]
\end{gathered}
$$

Assumindo em (12) a independência condicional. Assim, caso tenha-se uma amostra de indivíduos participantes do tratamento, $\mathrm{E}\left[y_{1} \mid D=1, x\right]$ pode ser estimada. Entretanto, $\mathrm{E}\left[y_{0} \mid x, D=0\right]$ não é observado, devido ser uma medida dos resultados médios para os participantes. Uma vez que, não é possível observar simultaneamente o mesmo indivíduo como participante e não participante. Logo, tem-se: 


$$
\begin{gathered}
\mathrm{ATE}=\mathrm{E}\left[y_{1} \mid x, D=1\right]-\mathrm{E}\left[y_{0} \mid x, D=0\right] \\
\mathrm{ATE}=\mu_{1}(x)-\mu_{0}(x)+E\left[u_{1} \mid x, D=1\right]-E\left[u_{0} \mid x, D=0\right] \\
\mathrm{ATE}=\mu_{1}(x)-\mu_{0}(x)+E\left[u_{1} \mid x\right]-E\left[u_{0} \mid x\right] \\
\mathrm{ATE}=\mu_{1}(x)-\mu_{0}(x)
\end{gathered}
$$

Onde o primeiro termo da Equação (13) pode ser estimado utilizando os dados dos participantes de tratamento, mas o segundo termo não é diretamente observável. Em (13), (14) e (15) segue-se assumindo independência condicional e média condicional. Além disso, adotam-se as especificações $y_{1}=\mu_{1}(x)+u_{1}$ para o tratado e $y_{0}=\mu_{0}(x)+u_{0}$ para o não tratado. Já o segundo termo do lado direito em (16) exige apenas a independência da média, em vez de independência condicional completa. Assim, portanto, apuram-se os resultados propostos em (7) e (8).

\subsection{Descrição dos Dados}

Faz-se agora uma leitura sintética da base de dados. Contudo, a priori apresentam-se no Quadro 1 as variáveis empregadas nesse estudo. Ressalta-se que, o conjunto de covariadas utilizadas foi selecionado obedecendo à literatura e alguns testes de especificação do modelo.

\begin{tabular}{|c|c|}
\hline Variáveis & Descrição \\
\hline Resultado Educacional & Logaritmo do Índice de Rendimento Acadêmico \\
\hline \multicolumn{2}{|r|}{ Variáveis de Circunstâncias } \\
\hline Educação Parental & Analfabeto $=1 ;$ Fundamental $=2 ;$ Médio $=3 ;$ Superior $=4$ \\
\hline Tipo de Escola & Dummy para escola. 0 para escola pública e 1 para privada. \\
\hline Sexo & Dummy para sexo. 0 para feminino e 1 para masculino. \\
\hline Atendimento Pré-escolar & Dummy para pré-escola. 0 para não atendidos e 1 para atendidos. \\
\hline Estrutura Familiar & $\begin{array}{l}\text { Dummy para estrutura familiar. } 0 \text { para monoparental e } 1 \text { para } \\
\text { biparental. }\end{array}$ \\
\hline Siblings - Irmãos & Número de irmãos. \\
\hline Renda Familiar & $\begin{array}{l}\text { Até } 1 \text { salário }=1 ; 1 \text { a } 3=2 ; 3 \text { a } 6=3 ; 6 \text { a } 9=4 ; \text { Acima de } 9 \text { salários } \\
=5 \text {. }\end{array}$ \\
\hline Cor & Dummy para cor. 0 para não brancos e 1 para brancos. \\
\hline \multicolumn{2}{|r|}{ Variáveis de Esforço Individual } \\
\hline Repetência & $\begin{array}{l}\text { Dummy para repetência. } 0 \text { caso já tenha reprovado e } 1 \text { caso } \\
\text { contrário. }\end{array}$ \\
\hline Migrante & Dummy para migrante. 0 caso seja migrante e 1 caso contrário. \\
\hline Horas de estudo semanal & 0 à $2 \mathrm{~h}=1 ; 2$ à $4 \mathrm{~h}=2 ; 4$ à $6 \mathrm{~h}=3 ; 6$ à $8 \mathrm{~h}=4 ; 8$ à $10=5 ;$ Acima de \\
\hline
\end{tabular}
Isto é, verificou-se através de testes econométricos a existência de variáveis omitidas, como também, a remoção ou adição de diversas variáveis nas estimações.

Quadro 1: Descrição das variáveis 
$10 \mathrm{~h}=6$.

Fonte: Elaboração Própria.

Posto isso, observa-se a partir da Tabela 1, que o rendimento educacional dos alunos da UFERSA - Pau dos Ferros - varia entre 2,7 a 9,0 com um desempenho médio situado em 6,3. Além disso, verifica-se que aproximadamente $2 \%$ dos alunos são filhos de mães analfabetas. No que se refere à educação do pai, constata-se que esse número salta para algo em torno de 9,6\%. Ou seja, o índice de pai analfabeto nas famílias dos estudantes é quase quatro vezes maior do observado para a mãe.

Essa assimetria educacional dos pais é constatada em praticamente todos os níveis educacionais, exceto no nível fundamental, onde se encontra um elevado nível de pais - homens - com esse patamar de educação (fundamental). Em números, cerca de $40 \%$ dos pais possuem esse grau de instrução. Corroborando com essa análise, verifica-se que as mães com nível superior representam quase o dobro do observado referente aos pais. Isto é, aproximadamente $27,1 \%$ das mães tem formação superior contra apenas 15,9\% dos pais.

Quanto à renda familiar, os dados informam que apenas 4,26\% das famílias tem renda superior a nove salários mínimos. Por outro lado, 13,3\% ganham apenas no máximo um salário. É importante destacar que praticamente metade dos estudantes analisados possui renda familiar entre um a três salários mínimos. Isto é, mais da metade da amostra possui origem familiar bastante simples.

Tabela 1: Estatística descritiva

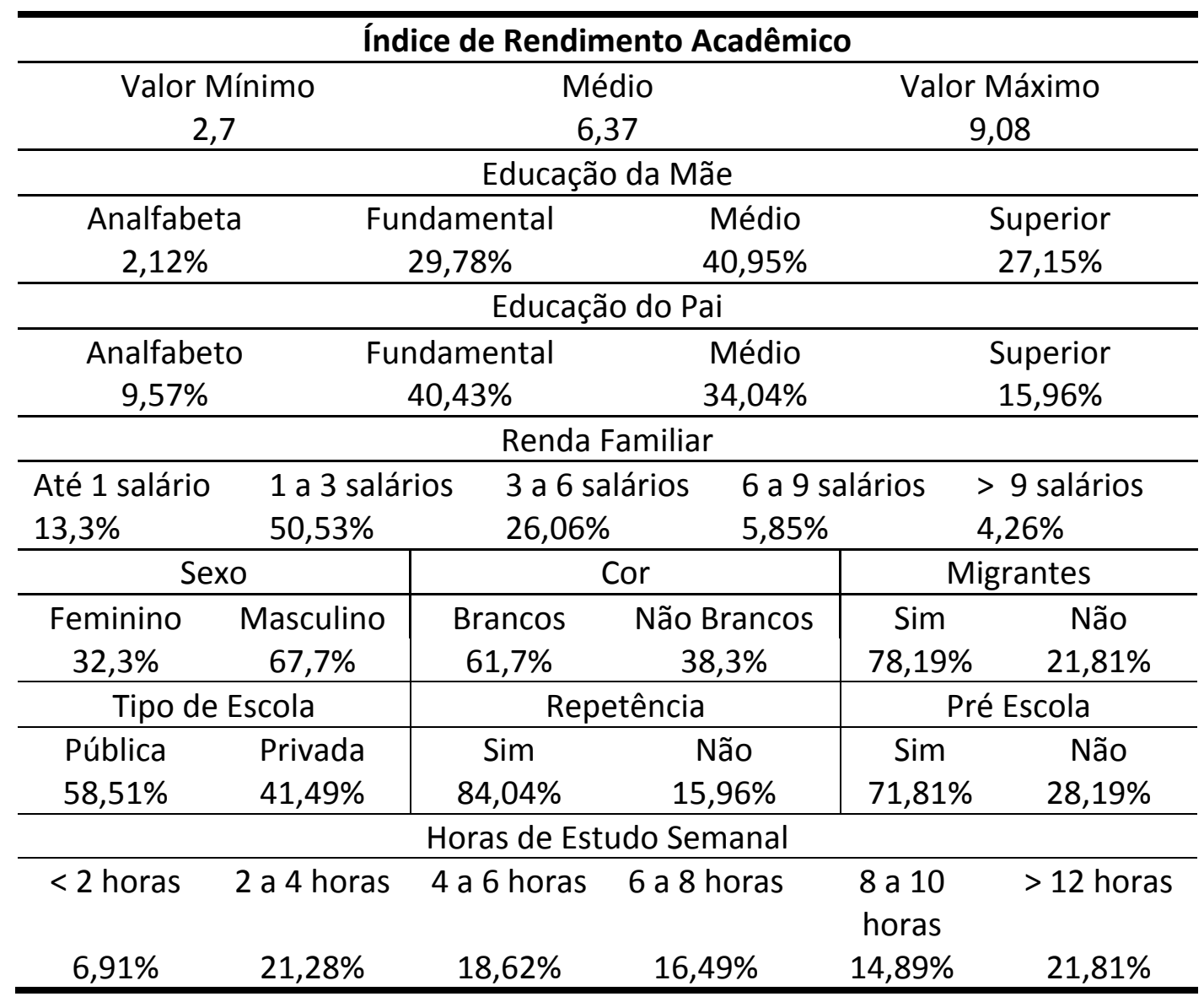

Fonte: Elaboração Própria. 
Outras características atribuídas ao conjunto de dados reportam que, aproximadamente $68 \%$ dos estudantes são do sexo masculino; $38 \%$ se declaram não brancos; $59 \%$ são oriundos de escola pública; $28 \%$ não frequentaram a pré-escola. Contudo, três características observadas nos dados merecem destaque: i) mais de $78 \%$ dos estudantes analisados são migrantes, isto é, são estudantes de outras localidades - municípios e/ou estados; ii) $84 \%$ dos alunos repetiram no mínimo uma vez alguma disciplina.

Esse dado por sua vez reflete um alto índice de reprovação nas disciplinas; iii) por fim, relata-se que, quase metade dos alunos estuda no máximo seis horas por semana. Essa última observação parece explicar os altos índices de reprovação e o baixo desempenho médio observado.

\section{RESULTADOS E DISCUSSÕES}

A priori, ilustra-se na Figura 1 a distribuição dos dados analisados. Aparentemente os dados se mostram normalmente distribuídos. Contudo, através do teste de normalidade verificou-se que os mesmos apresentam ruído branco. Posto isso, apresenta-se na Tabela 2 os resultados das estimações. A princípio destaca-se que todas as variáveis, exceto migração, são estatisticamente significativas. Porém, há outros resultados interessantes.

Dos fatores circunstanciais, isto é, as características as quais o indivíduo não possui qualquer controle sobre elas, como por exemplo, a formação educacional parental, reflete em $15,1 \%$ no rendimento estudantil. Todavia, ressalta-se que embora não se possa inferir que esses resultados representam causalidade, obteve-se um intervalo de confiança de $99 \%$, onde o impacto da educação parental varia entre $11,5 \%$ a $18,6 \%$ na composição do comportamento educacional para os alunos da UFERSA do campus Pau dos Ferros. Em outras palavras, pode-se afirmar que há fortes evidências que a educação dos pais contribui substancialmente para o sucesso educacional dos estudantes. Adicionalmente, destaca-se que este achado vai de encontro a estudos encontrados na literatura, por exemplo, em Figueiredo, Nogueira e Santana (2014); Foguel e Veloso (2014). 


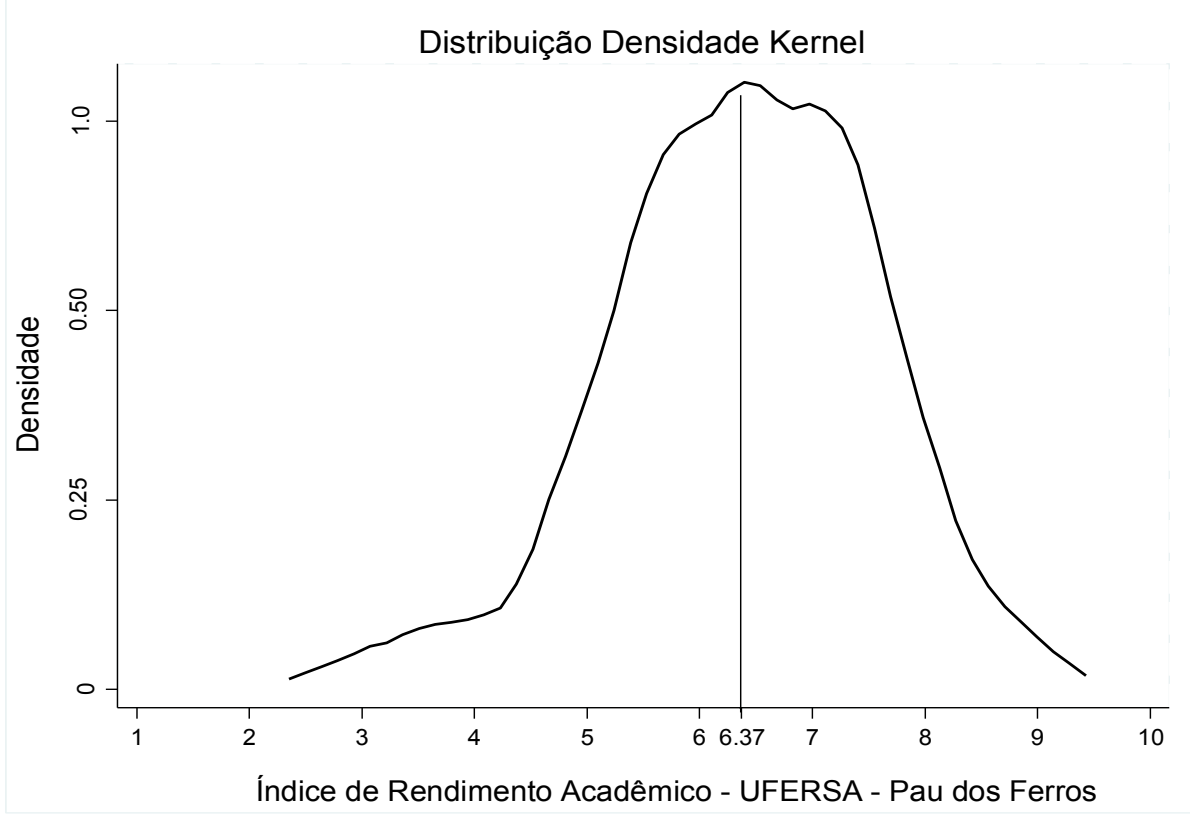

Figura 1: Distribuição do rendimento acadêmico.

Fonte: Elaboração própria.

Nesse contexto, outro importante atributo circunstancial apresenta-se como fator crucial no desempenho dos estudantes da instituição de ensino analisada. Isto é, a estrutura biparental - familiar influencia, em média, 14,9\% o rendimento acadêmico. Em outras palavras, os alunos que possuem uma estrutura familiar biparental apresenta forte correlação com a formação do desenvolvimento educacional. Outra característica que apresenta forte correlação com a variável de interesse - IRA - é a cor do estudante. Segundo os resultados obtidos, os estudantes declarados brancos tem um desempenho médio $15,6 \%$ superior comparado aos não brancos. Todavia, vale destacar que os alunos que declararam não brancos representam apenas $38 \%$ do total da amostra.

Por outro lado, no que se refere às características de responsabilidade, ou seja, aquelas as quais o indivíduo possui certo ou total controle sobre elas, por exemplo, reprovação e horas de estudo, influenciam respectivamente em $28 \%$ e $10,8 \%$. Esses resultados são esperados, uma vez que, há relatos empíricos que a reprovação exerce forte desestímulo no aluno. De forma análoga, é de se esperar que alunos mais estudiosos - maior esforço - adquiram mais conhecimentos, e, portanto, mais elevadas serão suas notas nas avaliações.

Tabela 2: Determinantes do índice de rendimento acadêmico

\begin{tabular}{l|l|c}
\hline Descrição & Coeficiente & Desvio Padrão \\
\hline Educação Parental & $0.1509 * * *$ & 0.0178 \\
Diferença Educacional & $0.1523 * * *$ & 0.0327 \\
Estrutura Familiar & $0.1489 * * *$ & 0.0512 \\
Renda & 0.0425 & 0.0320 \\
Sexo & $0.1105^{* *}$ & 0.0531 \\
Cor & $0.1562 * * *$ & 0.0519 \\
Pré-Escola & $0.1358^{* *}$ & 0.0555 \\
Tipo de Escola & $-0.1410^{*}$ & 0.0602 \\
Irmãos & $0.0490 * * *$ & 0.0130
\end{tabular}




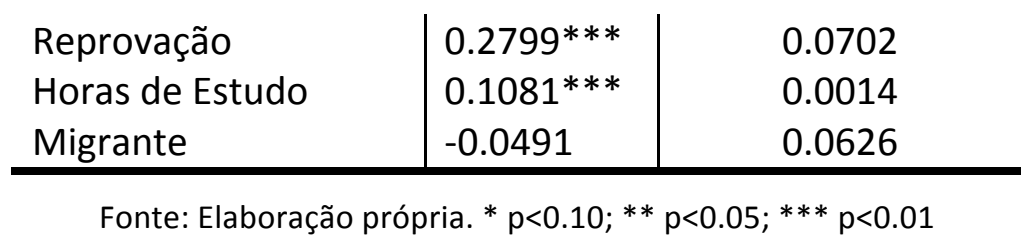

Um resultado bastante diferenciado é imputado à renda familiar. Pois, embora, apresente coeficiente positivo, o fator não se mostrou estatisticamente diferente de zero. Ou seja, possuir renda favorável não importa significativamente no resultado educacional quando comparado aos que possuem renda familiar mais vulnerável. Apesar dos dados disponíveis não possibilitar fazer indagações da razão desse resultado, acredita-se do papel crucial exercido pelas bolsas de assistência e mobilidade social na atenuação desta desigualdade. Percebe-se também, que os estudantes do sexo masculino em média tem nota superior em 0,11 , entretanto, essa característica representa $67 \%$ da amostra total o que talvez represente uma limitação da amostra.

Além disso, os alunos que são filhos únicos são impactados positivamente em 4,9\%. Contudo, há outros dois resultados que merecem destaque. O primeiro parece surpreender, pois, os resultados indicam que os alunos provenientes de escolas públicas são impactados em média em $14,1 \%$ na formação do rendimento escolar quando confrontados com alunos de escolas privadas. Segundo, não há evidencias empíricas sobre a influência do fator migração sobre o resultado individual. Presumisse que esses achados podem ser atribuídos, em especial, por duas particularidades respectivas: i) o viés de seleção, promovido pelo Sistema de Seleção Unificada SISU; e ii) o fato de mais de três quartos da amostra ser composta de migrantes.

Apesar dos resultados apurados se mostrarem interessantes, aplica-se a seguir duas estratégias com o propósito de enriquecer a análise: na primeira, segue-se O’Neill, Sweetman e van DeGaer (2001), isto é, adota-se uma abordagem não paramétrica, assumindo a RIA. Em síntese, admite total independência entre as variáveis de circunstâncias e de esforço individual. $\mathrm{Na}$ segunda abordagem, emprega-se uma modelagem de efeito tratamento médio seguindo Cameron e Trivedi (2005). Destaca-se que há duas finalidades essenciais no emprego desses exercícios complementares, em especial, a possível mensuração do esforço individual empregado em termos de probabilidade, quando separarmos os estudantes por tipos, ou seja, características familiares favoráveis ou não, também denominadas de sorte social ${ }^{4}$. O segundo exercício vale como teste de robustez, isto é, ver se os resultados se mantém quando adotamos uma estratégia mais rígida.

Nesse contexto, a Figura 2, permite desconfiar que os estudantes que possuem alto background social, a priori, tem um caminho menos íngreme em direção da média. Ou seja, os estudantes com baixo background social tem um percurso bem mais inclinado em direção à média do rendimento educacional. Contudo, por enquanto essa análise é apenas visual. Todavia, as estimações informam que a probabilidade de um aluno que possui baixo background social educação parental máxima com nível médio; renda familiar máxima de 3 salários e estrutura familiar monoparental - estar abaixo da nota média é de $75,2 \%$ contra $71,4 \%$ caso o mesmo possua alto background social - educação parental superior; renda familiar acima de 6 salários mínimos e possuir estrutura familiar biparental.

\footnotetext{
${ }^{4}$ Maiores detalhes em Vallentyne (2002).
} 


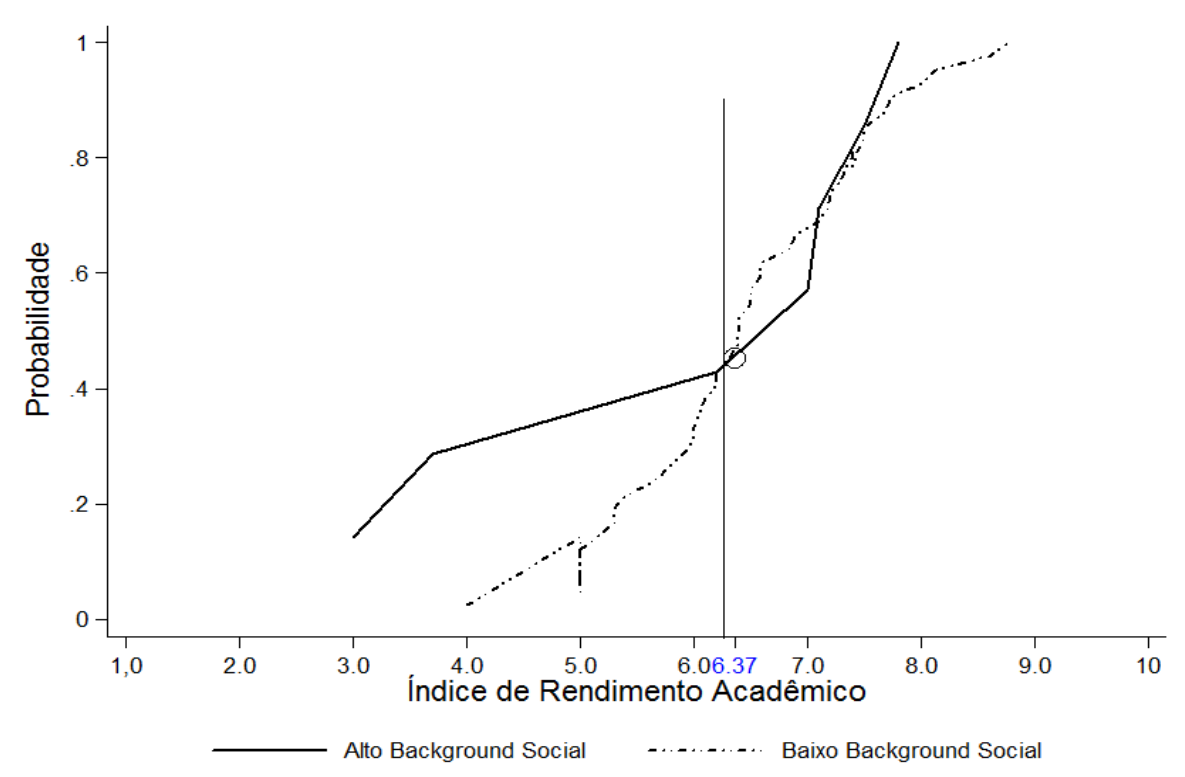

Figura 2: Esforço versus desempenho acadêmico

Fonte: Elaboração própria.

Em resumo, em termos de probabilidade, esse aluno tem que se esforçar 5,32\% a mais que o aluno com alto background social para não ficar abaixo da nota média. Analogamente, 0 aluno com alto background possui 1,053 vezes maiores chances de estar entre os que estão na média geral. Apesar disso, quando o assunto é estar acima da média, o cenário se inverte totalmente. Isto é, os alunos com fundo social desfavorável tem 15,5\% de pertencerem ao estrato com rendimento superior a sete. Para os com situação social inversa, essa probabilidade cai para $14,3 \%$. Em outras palavras, a chance de um aluno socialmente favorecido estar com um rendimento educacional acima de sete é $8,4 \%$ menor que o estudante com situação social oposta. A Figura 2 confirma visualmente essa análise.

Por sua vez, quando se verifica a probabilidade do estudante com baixo background social estar entre os seletos $6,3 \%$ melhores alunos da UFERSA, em termos de rendimento acadêmico, observa-se que as chances - probabilidade - destes estarem nessa faixa são de 3,6\%, contra $0 \%$ dos pertencentes ao tipo alto background social. Especificamente, dos 12 alunos que formam esse grupo - IRA maior ou igual a oito - nenhum deles tem pai com nível superior e apenas dois são filhos de mãe com esse grau de estudo.

Nessa linha de raciocínio, apenas um dos alunos nessa faixa de rendimento acadêmico tem renda familiar superior a seis salários mínimos. Adicionalmente, observa-se que metade é do sexo feminino e apenas $16,7 \%$ tem origem familiar em Pau dos Ferros. Além disso, verificou-se que $75 \%$ desse grupo de alunos estudam no mínimo oito horas por semana. Alguns desses fatos, em especial, o esforço individual - horas de estudo - parecem explicar o motivo pelo qual os alunos com situação social favorável não pertencem a esse distinto extrato educacional na UFERSA campus Pau dos Ferros.

Tabela 3: Efeito tratamento médio - circunstâncias e esforço 


\begin{tabular}{l|c|c}
\hline \multicolumn{1}{c|}{ Descrição } & Coeficiente & Desvio Padrão \\
\hline Educação Parental & 0.011 & 0.050 \\
Renda & 0.036 & 0.076 \\
Estrutura Familiar & -0.044 & 0.036 \\
Escola & -0.035 & 0.061 \\
Pré-escola & 0.002 & 0.045 \\
Horas de Estudo & $0.110 * * *$ & 0.043 \\
Repetência de Disciplina & $0.262 * * *$ & 0.042 \\
\hline
\end{tabular}

Fonte: Elaboração própria. * $p<0.10 ; * * p<0.05 ; * * * p<0.01$

Por fim, apresentam-se na Tabela 3, os resultados possibilitados com a terceira estratégia - Efeito Tratamento - adotada. Os mesmos apontam que somente pode-se afirmar que há fortes correlações nas covariadas de circunstâncias sociais, pois em nenhum desses fatores analisados foram encontradas evidências empíricas - efeito tratamento médio - significativas. Isto é, ao criar contrafatuais, por exemplo, se todos os alunos tivessem educação parental superior, renda variando acima de seis salários mínimos, estrutura familiar biparental, estudasse em escola pública, tivessem frequentado a pré-escola, e assim por diante, não são encontrados coeficientes estatisticamente diferentes de zero. Embora, os mesmos tenham se mostrados positivos, exceto, para o tipo de escola.

Porém, quanto o assunto são as variáveis de responsabilidade individual - esforço empreendido - os resultados se mostram cruciais segundo a metodologia de efeito tratamento. Especificamente, caso os alunos ingressassem num tratamento de estudar no mínimo seis horas por semana, em média, aumentaria a média em $11,1 \%$. Esse resultado demonstra a magnitude econômica - desempenho acadêmico - do impacto de esforço individual empregado. Parece estar-se sendo redundante, entretanto, é bastante atraente encontrar evidências empíricas que estudar mais é a chave para o sucesso do aluno.

Nessa perspectiva, outro resultado bastante interessante refere-se à questão da repetência de disciplina. As estimações revelam um impacto negativo de $26 \%$ da reprovação de ao menos uma disciplina na composição do rendimento acadêmico. Sinteticamente, isso significa que, caso os alunos reprovados em pelo menos uma disciplina não tivesse passado por tal fato, sua média evoluiria positivamente em $26 \%$. Esse resultado se mostra bastante plausível, uma vez que, a reprovação na maior parte das vezes tem um efeito desestimulador enorme.

Destaca-se que não foram criados contrafatuais para a variável diferença educacional dos pais, sexo, cor, número de irmãos e migração. Dado que, quando se constrói um contrafatual para todos os pais com mesma formação educacional - nível superior - não há mais diferenças nesse sentido. A variável migração ficou de fora da metodologia em função de não apresentar valores significativos estatisticamente na primeira abordagem. Quanto as variável sexo, cor e número de irmãos são imutáveis, pois não se refere a condições expostas pela sociedade.

\section{CONCLUSÃO}

Este estudo buscou promover uma ampla investigação sobre o processo de formação do Índice de Rendimento Acadêmico dos alunos da UFERSA - campus Pau dos Ferros. Em síntese, o 
estudo tem como principal objetivo mensurar os principais determinantes desse índice. Para tanto, construiu-se uma base de dados a partir de uma pesquisa socioeconômica com os alunos do campus. Adicionalmente, adotaram-se três estratégias empíricas a fim de atingir os objetivos propostos.

Os principais resultados informam a existência de impactos - correlação - de variáveis circunstanciais como: educação parental, assimetria educacional parental, estrutura familiar, sexo, cor, tipo de escola, pré-escola, número de irmãos. No que se refere às variáveis de esforço individual, como horas de estudo e reprovação de disciplinas foram encontradas evidências de causa. Em outras palavras, estudar mais e não ter sido reprovado, em média, tem efeito substancial no desempenho educacional. Adicionalmente, ao utilizar a segunda metodologia, percebe-se uma dificuldade dos alunos com baixo background social familiar em atingir a nota média, contudo, a partir desse momento, verifica-se uma maior chance dos alunos pertencentes a esse extrato social estar entre os melhores. Especificamente, os doze alunos com média igual ou superior a oito pertencem a essa condição social.

Nesse sentido, ao menos três inferências podem ser destacadas: i) parece haver participação significativa das condições sociais no desempenho educacional; ii) é possível verificar o diferencial de esforço necessário para o aluno com baixo background social atingir a média, e a partir dessa, o esforço parece dominar consideravelmente as condições sociais desfavoráveis; e, por fim iii) há evidências de efeito tratamento para as variáveis de esforço. Esse último resultado é corroborado pelas duas metodologias anteriores.

\section{REFERÊNCIAS}

Figueiredo, E. A. D., Santos, D. F. S. W. P., \& Annegues, A. C. (2015). Injusticia de la desigualdad: factores determinantes en el Brasil, 1995 y 2009. Revista CEPAL

Arneson, R. J. (1989). Equality and equal opportunity for welfare. Philosophical studies, 56(1), 7793.

Ashenfelter, O., \& Rouse, C. (1998). Income, schooling, and ability: Evidence from a new sample of identical twins. The Quarterly Journal of Economics, 113(1), 253-284.

Black, S. E., Devereux, P. J., \& Salvanes, K. G. (2005). The more the merrier? The effect of family size and birth order on children's education. The Quarterly Journal of Economics, 120(2), 669700.

BOURGUIGNON, F.; FERREIRA, F.; MILANOVIC, B.; RAVALLION, M. Global Inequality. in Kenneth ReinertandRamkishenRajan (eds) Princeton Encyclopediaofthe World Economy, Princeton University Press, forthcoming, 2007.

Cameron, A. C., \& Trivedi, P. K. (2005). Microeconometrics: methods and applications. Cambridge university press.

Daude, C. (2011). Ascendance by descendants? On intergenerational education mobility in Latin America. OECD Development Centre Working Papers, (297), 1.

Diaz, M. D. M. (2012). (Des) Igualdades de Oportunidades no Ensino Médio Brasileiro: Escolas Públicas e Privadas. Economia, 13(3a).

Dworkin, R. (1981). What is equality? Part 2: Equality of resources. Philosophy \& Public Affairs, 283-345. 
Ferreira, F. H., \& Gignoux, J. (2011). The measurement of inequality of opportunity: Theory and an application to Latin America. Review of Income and Wealth, 57(4), 622-657.

Figueirêdo, E., Nogueiray, L., \& Santanaz, F. L. (2014). Igualdade de Oportunidades: Analisando o papel das circunstâncias no desempenho do ENEM. Revista Brasileira de Economia, 68(3), 373392.

Firpo, S., Ponczek, V. P., \& Possebom, V. (2014). Private Education Market, Information on Test Scores and Tuition Practices.

Fleurbaey, M. (1995). Equal opportunity or equal social outcome?. Economics and philosophy, 11(01), 25-55.

Foguel, M. N., \& Veloso, F. A. (2014). Inequality of opportunity in daycare and preschool services in Brazil. Journal of Economic Inequality, 12(2), 191.

Hanushek, E. A. (1979). Conceptual and empirical issues in the estimation of educational production functions. Journal of human Resources, 351-388.

Hanushek, E. (1970). The Production of Education, Teacher Quality and Efficiency.

Junior, J. L. D. S. N., de Brito Ramalho, H. M., \& da Silva, E. K. (2013). Transmissão Intergeracional de educação e mobilidade de renda no Brasil. Revista Economia e Desenvolvimento, 12(2).

Klein, R., \& Vella, F. (2010). Estimating a class of triangular simultaneous equations models without exclusion restrictions. Journal of Econometrics, 154(2), 154-164.

Leite, Á. R., Justo, W. R., \& Júnior, J. L. D. S. N. Análise dos Principais Determinantes da Mobilidade Intergeracional de Educação entre as Regiões Nordeste e Sudeste.

Nogueira, L. C. B., \& de Alencar, E. F. (2015). Tal Pai, Tal Filho? Uma Análise dos Efeitos Fatores de Circunstâncias Sobre o Desempenho dos Alunos na Avaliação do PISA 2012. Texto para discussão. Universidade Federal da Paraíba.

John, R. (1971). A theory of justice.

Roemer, J. E., \& Trannoy, A. (1998). Equality of Opportunity.

Romanello, M., Sawyer, D. O., De Oliveria, F. G. .Youth Informality In Brazil: An Analysis Of SchoolTo-Work Transition. 2013.

Vallentyne, P. (2002). Brute luck, option luck, and equality of initial opportunities. Ethics, 112(3), 529-557. 